\title{
Measurement of static stress in round bar by impact sound
}

${ }^{\text {T. Yoshida }}{ }^{1}$, K. Sakurada ${ }^{1}$ and M. Hoshino

'Mech. Systems Eng., Faculty of Eng., Takushoku Univ., Tatemachi 815-1, Hachioji, Tokyo, Japan.

\section{Introduction}

We have been proposing the method by an impact sound to measure Young's moduli of an anisotropic material [1], or one between $-150 \sim 1300$ [2]. In this research, we applied the method to measure static working stress in a round bar and examined the validity.

\section{Natural frequencies of round bar subjected to axial load}

A vibration equation of the bar subjected to an axial load, $P$, is given as the next expression (1).

$$
\frac{\partial^{2} y}{\partial t^{2}}+\frac{E I_{g}}{w A} \frac{\partial^{4} y}{\partial x^{4}}+\frac{g P}{w A} \frac{\partial^{2} y}{\partial x^{2}}=0
$$

Provided with $y$ as deflection of the bar, $x$ and $t$ as location and time variables respectively, $I$ as sectional secondary moment, $g$ as acceleration of gravity, $w$ as weight per unit volume and $A$ as area. When we suppose the deflection of the bar as $y=X(x)^{*} e^{\text {int }}$, provided with $n$ is a natural angular frequency and $i$ is imaginary number unit. Natural vibration deformation mode, $X$ is expressed as (2).

$$
X=A \cos \lambda_{1} x+B \sin \lambda_{1} x+C \cosh \lambda_{2} x+D \sinh \lambda_{2} x
$$

As a boundary condition, both ends of the bar were fixed. A determinant of the coefficient matrix of expression (2) applied with the boundary condition must be zero. The determinant is the function of load $P$, frequency $n$ and the material property values. Designating certain value of load $P$ and the property values, we tried to find frequency values which made the determinant to be zero. The frequency is one of natural frequencies which consist of the impact sound with the bar.

Schematic experiment system employed in this research is shown in Figure 1. A small steel sphere with $3 \mathrm{~mm}$ diameter is dropped onto a round bar. The bar is applied with an axial load and its both ends are fixed by an apparatus. The impact sound occurred in the collision between the bar and the sphere ball is measured by microphone. Sound pressure variation data is analyzed using FFT equipment and natural frequencies were evaluated. Working stresses were measured by the strain gages attached on the bar. We used the bar with $200 \mathrm{~mm}$ long and two kinds of diameter, $6 \mathrm{~mm}$ and 8 $\mathrm{mm}$. The experimental results were compared with those by the theoretical analysis explained above

\section{Result}

Figure 2 shows the comparison between the theoretical and experimental results of the 1st mode with 8 pround bar. Horizontal axis is the working stress measured by the strain gages in the experiment. Vertical axis is the natural frequency evaluated by FFT analysis for the impact sound. Theoretical relation between natural frequency and working stress is shown by solid line. For the

This is an Open Access article distributed under the terms of the Creative Commons Attribution-Noncommercial License 3.0, which permits unrestricted use, distribution, and reproduction in any noncommercial medium, provided the original work is properly cited. 
Bull e

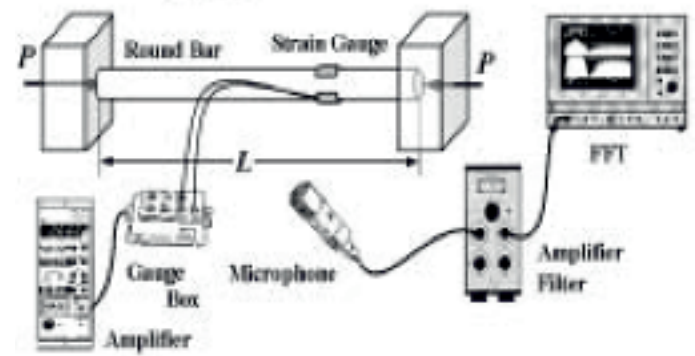

Fig. 1 Impact Sound Measurement System.

calculation, Young's modulus, $206 \mathrm{GPa}$ and density, $7.98 \mathrm{~g} / \mathrm{cm}^{3}$ were used.

Figure 2 shows that the larger working stress (tensile load) is applied, the higher the natural frequency goes up. The smaller working stress (compression load) is applied, the lower the natural frequency goes down. There is about $5 \%$ difference between the theoretical and experimental results, but their gradients agreed well. This suggests us how we measure working stress in a bar employing an impact sound. First we measure a natural frequency of a bar with no load experimentally. Second we draw the line with the theoretical gradient which passes the above experimental frequency with no load. The line enables us to measure working stress magnitude in a bar by Fig.2 Relation between applied stress measuring natural frequency of impact sound and natural frequency

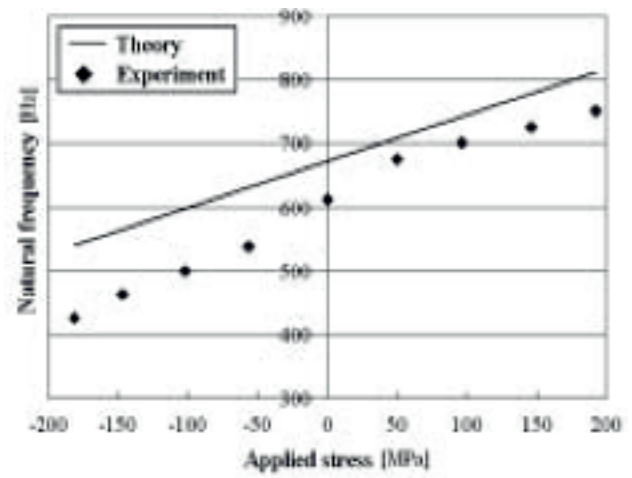

\section{Conclusion}

A round bar with both rigid ends was subjected to tensile or compression stress and impacted by a small ball. By FFT analyzer, natural frequencies of the bar were measured employing the impact sound. Working stress in the bar was measured by strain gauges attached on the bar. Vibration equation for a bar with both rigid ends and subjected to an axial stress was analyzed and theoretical relation between applied stress and natural frequencies was obtained. The relation between applied stress and natural frequencies of the round bar by the experiment and by the theory was compared. The experimental and the theoretical results agreed with about $5 \%$ difference. Impact sound may be one of effective methods to measure working stress in a bar subjected to an axial stress with ease and high reliability.

\section{References}

1. T.Yoshida, S.Matsui, M.Hoshino, M.Sakata, 5th Int. Conf. on Fracture \& Strength of Solids (2003)

2. M.Sakata, H.Ohnabe, Proc. of American Society for Composites 9th Tech. Conf. (1994) 